A. Dias, D. Bouvier, S. Cusack,

R. W. H. Ruigrok, T. Crépin : Biologie

structurale des interactions entre virus

\title{
Nouvelle stratégie pour cibler la réplication du virus de la grippe
}

Alexandre Dias, Denis Bouvier, Stephen Cusack, Rob W.H. Ruigrok, Thibaut Crépin et cellule hôte, UMR 5233 UJF, EMBL, CNRS, 6, rue Jules Horowitz, BP181, 38042 Grenoble Cedex 9, France

S. Cusack : Laboratoire Européen de Biologie Moléculaire, antenne de Grenoble, 6, rue Jules Horowitz, BP181, 38042 Grenoble Cedex 9, France. crepin@embl.fr dias@embl.fr

> La grippe est une maladie saisonnière occasionnant chaque année le décès de plusieurs centaines de milliers de personnes dans le monde. De plus, l'émergence de nouvelles souches grippales entraîne périodiquement des pandémies dévastatrices, comme ce fût le cas en 1918 lorsque la grippe provoqua la mort de plusieurs dizaines de millions d'individus. L'apparition de la souche aviaire $\mathrm{H} 5 \mathrm{Nl}$ et sa transmission épisodique à l'homme mettent actuellement en exergue la nécessité de développer de nouvelles molécules antivirales.

\section{Le vol de coiffe}

Pour se répliquer et proliférer, le virus de la grippe doit détourner plusieurs processus clés de la cellule hôte. L'ARN polymérase ARN-dépendante du virus, complexe hétéro-trimérique composé des sous-unités PB1, PB2 et PA (Figure 2A), est responsable de la réplication et de la transcription de l'ARN viral dans le noyau des cellules infectées. Le complexe trimérique initie la transcription de l'ARN viral par le biais d'un mécanisme hautement spécifique dénommé «vol de coiffe» (cap-snatching en anglais). Au cours de ce processus, I'ARN polymérase virale cible un signal chimique primordial pour initier la traduction du génome cellulaire en protéines, la coiffe des ARN messagers (ARNm) cellulaires. La coiffe est en effet l'élément indispensable aux ARNm pour être reconnus et traduits par les
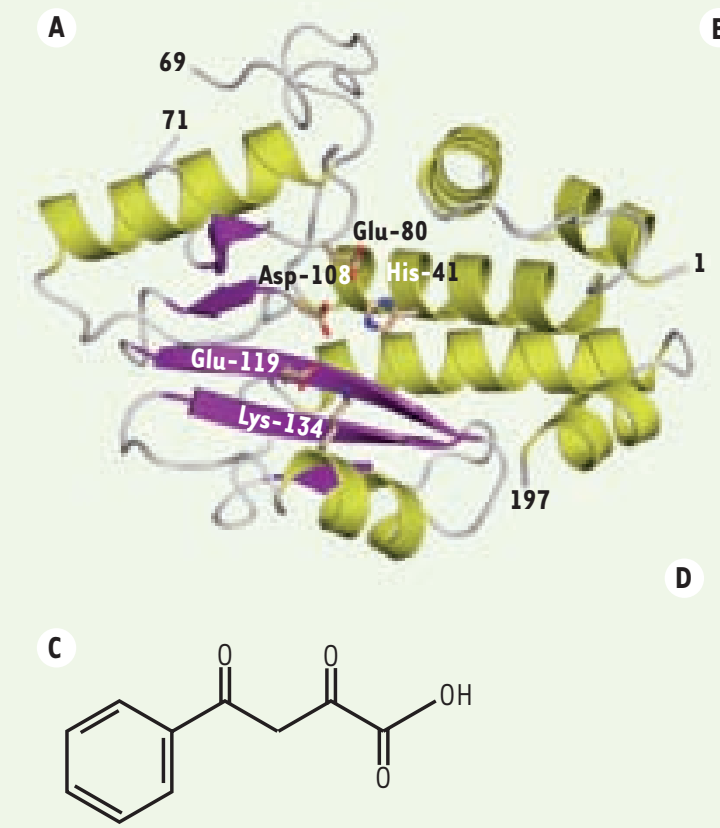

B

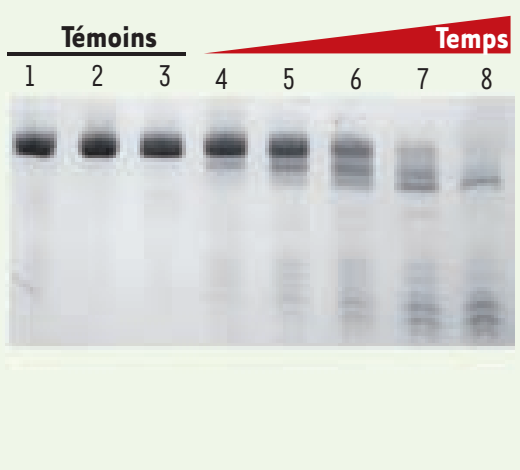

D

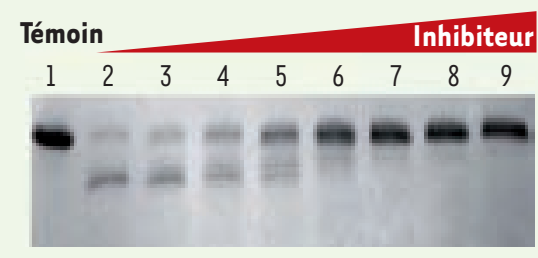

Figure 1. L'activité endonucléase de l'ARN polymérase du virus de la grippe est portée par le domaine PA-Nter. A. Structure tridimensionnelle du domaine PA-Nter. Les résidus formant le site actif sont annotés en fonction de leur position. B. Activité endonucléase manganèse-dépendante $d u$ domaine PA-Nter. Pour toutes les lignes, les concentrations en ARN, en protéine (PA-Nter) et en manganèse sont constantes, seul le temps de réaction varie entre les lignes $\mathbf{4}$ et $\mathbf{8}$ (respectivement $5,10,20,40$ et 80 minutes). La réaction

est arrêtée par l'ajout d'EGTA (ethylene glycol tetraacetic acid) à une concentration finale de 1 mM. La ligne $\mathbf{l}$ correspond à l'ARN mis en présence de PA-Nter seul, la 2, I'ARN en présence de manganèse seul, la 3, l'ARN en présence de PA-Nter + manganèse + EGTA. C. Formule topologique de l'acide 2,4 dioxo-4-phénylbutanoïque. $D$. Inhibition de l'activité endonucléase de PA-Nter par l'acide 2,4 dioxo-4-phénylbutanoïque. Les concentrations du domaine PA-Nter, de l'ARN et du manganèse restent constantes. Les réactions se déroulent sur 40 minutes, seule la concentration en inhibiteur augmente entre la ligne 2 et la ligne 9 (respectivement 0, 6.5, 13, 20, 26, 40, 65, 130 M). Un témoin de digestion a été réalisé en incubant l'ARN avec $1 \mathrm{mM}$ de manganèse (ligne $\mathbf{1}$ ). 
ribosomes. Le virus de la grippe est incapable de produire ses propres ARNm coiffés. Sa stratégie consiste donc à récupérer la coiffe des pré-ARNm cellulaires présents dans le noyau en les excisant pour initier la transcription de son propre génome. Bien que sujet à discussion, ce mécanisme de vol de la coiffe a finalement fait l'objet d'un consensus [1]. La sous-unité PB2 lierait de manière spécifique, par un site de fixation que notre laboratoire a réussi à caractériser [2], la coiffe des pré-ARNm cellulaires. L'ARNm serait ensuite clivé 10-13 nucléotides en aval de la coiffe par une activité endonucléase [1], qui fut un temps associée à la sous-unité PB2 [3]
A

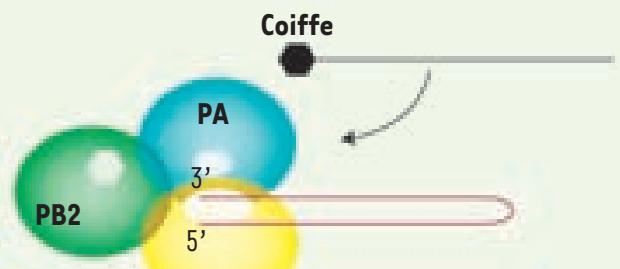

PB1

B

C

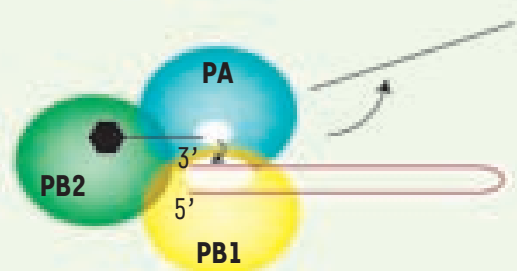

D

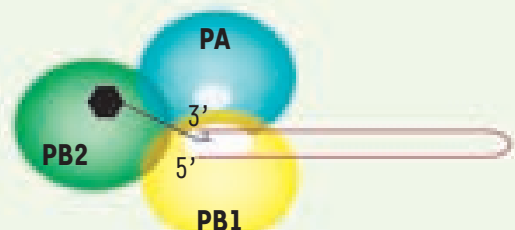

$\varepsilon$

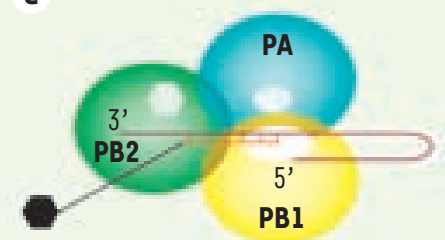

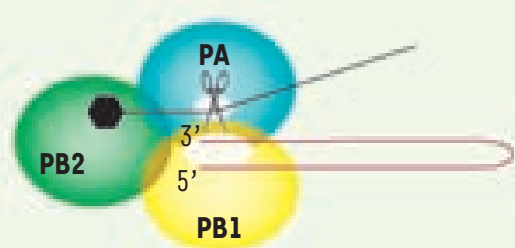

puis à la sous-unité PBI [4]. Ce morceau d'ARN coiffé serait subséquemment utilisé en tant qu'amorce par la sous-unité PBl pour initier la transcription de l'ARN viral. Ce schéma ne suggérait pas jusqu'à présent de rôle précisément décrit pour la sous-unité PA.

\section{Rôle de la sous unité PA dans le vol de la coiffe}

Nos études structurales et biochimiques [5] établissent pour la première fois un rôle fondamental pour la sous-unité PA dans le processus de vol de la coiffe, contredisant la vision généralement admise selon laquelle la sous-unité PBI abrite l'activité endonucléase. Nous avons pu obtenir, purifier et caractériser le domaine aminoterminal de la sous-unité PA (PA-Nter). Des cristaux du domaine ont été obtenus à partir desquels nous avons résolu la structure tridimensionnelle de PANter par diffraction des rayons $X$. L'obtention de cette structure à haute résolution nous a alors permis d'identifier une similitude structurale avec le site actif de certaines nucléases, notamment $\varepsilon \mathrm{coRV}$, enzyme de restriction de type II [6]. Les résidus His-41, Asp-108, Glu-119 et Lys-134 de PANter (Figure lA) se superposent à ceux du site actif de EcoRV. PA-Nter présente également une séquence similaire à celle de la superfamille des nucléases comportant un motif PD-(D/E)XK. Toutefois, la distance séparant les deux résidus acides (acide aspartique, D) du motif PDLYDYK de PA-Nter est inhabituellement courte. De plus, la lysine «catalytique » (Lys-134) [7] a migré à une autre position dans la séquence primaire de la protéine. Enfin, la structure tridimensionnelle souligne la présence, au sein du domaine, de deux atomes de manganèse coordonnés de manière similaire à ceux présents dans la structure de EcoRV [6].

En plus de l'identification de sa structure proche de celle de certaines nucléases, nous avons montré que PA-Nter possède également une activité endonucléase dépendante de la présence de manganèse (Figure 1B). Ces résultats corroborent parfaitement les données collectées sur l'activité endonucléase de l'ARN polymérase virale à ce jour [8]. L'activité endonucléase de PA-Nter a été étudiée en détail et nous a permis de tester des molécules inhibitrices. L'acide 2,4-dioxo4-phenylbutanoïque (Figure 1C) fait partie de ces molécules [9], et son action inhibitrice dose-dépendante sur l'activité nucléase du domaine PA-Nter a pu clairement être établie (Figure 1D).

\section{Un nouveau scénario \\ pour le vol de la coiffe}

Nos données concernant le domaine PANter, combinées aux données déterminées précédemment par notre laboratoire pour le domaine de liaison de la coiffe de la sousunité PB2 [2], nous permettent de proposer un nouveau schéma pour le mécanisme du vol de la coiffe dans lequel la sous-unité PA est largement impliquée (Figure 2). La sousunité PB2 lierait spécifiquement, via son domaine de fixation, la coiffe des pré-ARNm cellulaires (Figure 2A). Cela permettrait de placer l'ARNm au niveau du site actif de l'endonucléase virale, que nous avons identifiée comme étant portée par le domaine amino-
Figure 2. Mécanisme de vol de la coiffe. A. L'ARN polymérase du virus de la grippe fixe spécifiquement la coiffe d'un pré-ARNm cellulaire à l'aide du domaine de liaison de la coiffe porté par la sous-unité PB2. B. L'ARNm est clivé par le site endonucléase porté par la sous-unité PA. C. L'ARNm coupé est relargué par l'ARN polymérase virale alors que le morceau d'ARN coiffé bascule vers le site catalytique de la sous-unité PBI. D. Initiation de la transcription de l'ARNm viral à partir du morceau d'ARN coiffé. $\varepsilon$. દ́longation du transcrit viral par la sous-unité $\mathrm{PBl}$. 
terminal de la sous unité PA. PA-Nter cliverait I'ARNm (Figure $2 B$ ). II en résulterait un basculement du morceau d'ARNm coiffé vers la sous-unité PBI (Figure 2C) qui utiliserait ce fragment pour amorcer la transcription des ARNm viraux (Figures 2D et $2 \varepsilon$ ). Après leur transport du noyau vers le cytoplasme, ces ARNm viraux seraient alors reconnus par les ribosomes de la cellule infectée comme des ARNm endogènes et traduits en protéines.

\section{Bloquer le vol de la coiffe :}

\section{une piste thérapeutique prometteuse}

Notre étude du domaine PA-Nter a également mis en évidence que l'activité nucléase pouvait être inhibée par l'acide 2,4dioxo-4-phénylbutanoïque de manière dose dépendante. Compte tenu de la conservation des résidus du site actif entre les différentes souches virales, cette découverte ouvre de nouvelles perspectives quant à la concep- tion de molécules antivirales et fait de PA-Nter une cible de choix pour bloquer le processus de réplication du virus de la grippe dans les cellules. La structure tridimensionnelle à haute résolution de PA-Nter que nous avons obtenue sera alors d'une grande utilité pour concevoir et sélectionner des inhibiteurs rationnellement conçus, spécifiques de I'ARN polymérase du virus de la grippe. $\diamond$ New strategy for targeting influenza virus replication

\section{REMERCIEMENTS}

Ce travail a été partiellement financé par le contrat de l'union européenne FLUPOL (SP5BCT-2007-044263), le contrat ANR FLU INTERPOL (ANR-06-MIME-014-02) et Lyon Biopôle.

\section{RÉFÉRENCES}

1. Rao P, Yuan W, Krug RM. Crucial role of CA cleavage sites in the cap-snatching mechanism for initiating viral mRNA synthesis. EMBO / 2003; 22 : 1188-9.
2. Guilligay D, Tarendeau F, Resa-Infante P, et al. The structural basis for cap binding by influenza virus polymerase subunit PB2. Nat Struct Mol Biol $2008 ; 15: 500-6$.

3. Shi L, Summers DF, Peng 0 , Galarz JM. Influenza A virus RNA polymerase subunit $\mathrm{PB} 2$ is the endonuclease which cleaves host cell mRNA and functions only as the trimeric enzyme. Virology 1995 ; 208 : 38-47.

4. Li ML, Rao P, Krug RM. The active sites of the influenza cap-dependent endonuclease are on different polymerase subunits. EMBO / 2001; 20 : 2078-86.

5. Dias A, Bouvier D, Crépin T, et al. The cap-snatching endonuclease of influenza virus polymerase resides in the PA subunit. Nature 2009, 4 février (online).

6. Horton NC, Perona JJ. DNA cleavage by EcoRV endonuclease: two metal ions in three metal ion binding sites. Biochemistry $2004 ; 43$ : 6841-57.

7. Hara K, Schmidt FI, Crow M, Brownlee GG. Amino acid residues in the $\mathrm{N}$-terminal region of the $\mathrm{PA}$ subunit of influenza A virus RNA polymerase play a critical role in protein stability, endonuclease activity, cap binding, and virion RNA promoter binding. J Virol 2006 ; 80 : 7789-98.

8. Doan L, Handa B, Roberts NA, Klumpp K. Metal ion catalysis of RNA cleavage by the influenza virus endonuclease. Biochemistry 1996; $38: 5612-9$

9. Tomassini J, Selnick H, Davies ME, et al. Inhibition of cap ( $\mathrm{m} 7 \mathrm{GpppXm}$ )-dependent endonuclease of influenza virus by 4-substituted 2,4-dioxobutanoic acid compounds. Antimicrob Agents Chemother 1994 ; 38 : 2827-37.

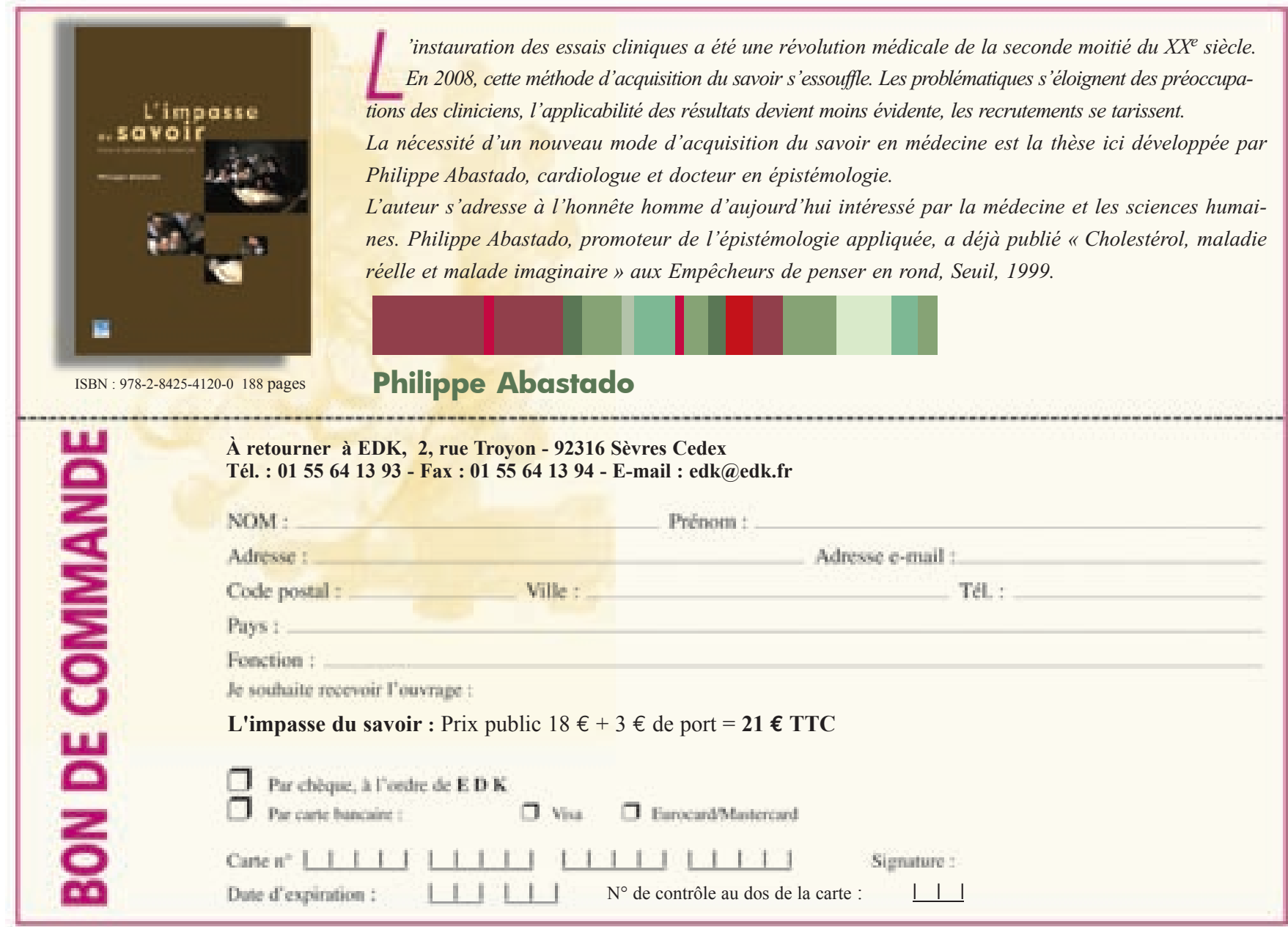

